\title{
Induction of autophagy-dependent necroptosis is required for childhood acute lymphoblastic leukemia cells to overcome glucocorticoid resistance
}

\author{
Laura Bonapace, ${ }^{1}$ Beat C. Bornhauser, ${ }^{1}$ Maike Schmitz, ${ }^{1}$ Gunnar Cario, ${ }^{2}$ Urs Ziegler, ${ }^{3}$
}

Felix K. Niggli, ${ }^{1}$ Beat W. Schäfer, ${ }^{1}$ Martin Schrappe, ${ }^{2}$ Martin Stanulla, ${ }^{2}$ and Jean-Pierre Bourquin ${ }^{1}$

1Department of Oncology, University Children's Hospital, University of Zurich, Switzerland. 2Department of Pediatrics, University Hospital Schleswig Holstein, Kiel, Germany. ${ }^{3}$ Center for Microscopy and Image Analysis, University of Zurich.

\begin{abstract}
In vivo resistance to first-line chemotherapy, including to glucocorticoids, is a strong predictor of poor outcome in children with acute lymphoblastic leukemia (ALL). Modulation of cell death regulators represents an attractive strategy for subverting such drug resistance. Here we report complete resensitization of multidrug-resistant childhood ALL cells to glucocorticoids and other cytotoxic agents with subcytotoxic concentrations of obatoclax, a putative antagonist of BCL-2 family members. The reversal of glucocorticoid resistance occurred through rapid activation of autophagy-dependent necroptosis, which bypassed the block in mitochondrial apoptosis. This effect was associated with dissociation of the autophagy inducer beclin- 1 from the antiapoptotic BCL-2 family member myeloid cell leukemia sequence 1 (MCL-1) and with a marked decrease in mammalian target of rapamycin (mTOR) activity. Consistent with a protective role for mTOR in glucocorticoid resistance in childhood ALL, combination of rapamycin with the glucocorticoid dexamethasone triggered autophagy-dependent cell death, with characteristic features of necroptosis. Execution of cell death, but not induction of autophagy, was strictly dependent on expression of receptor-interacting protein (RIP-1) kinase and cylindromatosis (turban tumor syndrome) (CYLD), two key regulators of necroptosis. Accordingly, both inhibition of RIP-1 and interference with CYLD restored glucocorticoid resistance completely. Together with evidence for a chemosensitizing activity of obatoclax in vivo, our data provide a compelling rationale for clinical translation of this pharmacological approach into treatments for patients with refractory ALL.
\end{abstract}

\section{Introduction}

Resistance to the initial phase of chemotherapy, in particular poor response to glucocorticoids (GCs), is a strong predictor of adverse outcome for childhood acute lymphoblastic leukemia (ALL) $(1,2)$. During the last decade, the cooperative Berlin-Frankfurt-Muenster (BFM) study group has validated an effective risk stratification approach, which is based on the assessment of the in vivo response to chemotherapy by leukemia-specific quantitative PCR. A group of patients at very high risk for relapse (VHR-ALL) can be identified based on persistence of minimal residual disease (MRD) (3). Because this is likely to reflect de novo resistance to multiple conventional antileukemic agents, combination treatment with new agents that modulate regulators of cell death represents an attractive approach to improve treatment response.

In GC-resistant ALL, the mechanisms underlying defective induction of mitochondrial apoptosis are still not understood. Increased expression of antiapoptotic myeloid cell leukemia sequence 1 (MCL-1) was a predominant feature of the gene expression signature of GC resistance (4). A bioinformatic screen of drugassociated signatures identified rapamycin as a sensitizer to GC

Authorship note: Laura Bonapace and Beat C. Bornhauser contributed equally to this work.

Conflict of interest: The authors have declared that no conflict of interest exists. Citation for this article: J Clin Invest. 2010;120(4):1310-1323. doi:10.1172/JCI39987. drugs in GC-resistant ALL. The GC-sensitizing effect of rapamycin was attributed to a decrease in MCL-1 levels, which was proposed to decrease the threshold to apoptotic stimuli by GC drugs (5).

Based on these considerations, we sought to evaluate the potential of the small molecule obatoclax (GX15-070) for combination therapy in refractory childhood ALL. This agent was proposed to act as a BCL-2 family antagonist and to disrupt the interaction between MCL-1 and its proapoptotic counterparts at cytotoxic concentrations $(6,7)$. Obatoclax was shown to trigger apoptosis at concentrations that resulted in disruption of Bak from MCL-1 and cytochrome $c$ release. However, obatoclax was also cytotoxic in cells that are deficient for the apoptosis effectors BAX and BAK, and lower concentrations of compound were sufficient to inhibit clonogenic growth of AML cells, suggesting the existence of additional target mechanisms (8). A recommended phase II dose has been established for adult patients with hematologic malignancies with an acceptable toxicity profile $(9,10)$, which constitutes the basis for further evaluation of obatoclax in pediatric trials.

In the context of defective apoptosis, an alternative cell death pathway has been identified that is dependent on macroautophagy $(11,12)$, a major form of autophagy, in which parts of the cytoplasm and intracellular organelles are sequestered within characteristic double-membraned or multi-membraned autophagic vacuoles (hereafter referred to as autophagy) (13). Autophagy is usually triggered to respond to increased metabolic requirements at times of cellular stress. Selected antiapoptotic BCL-2 
A
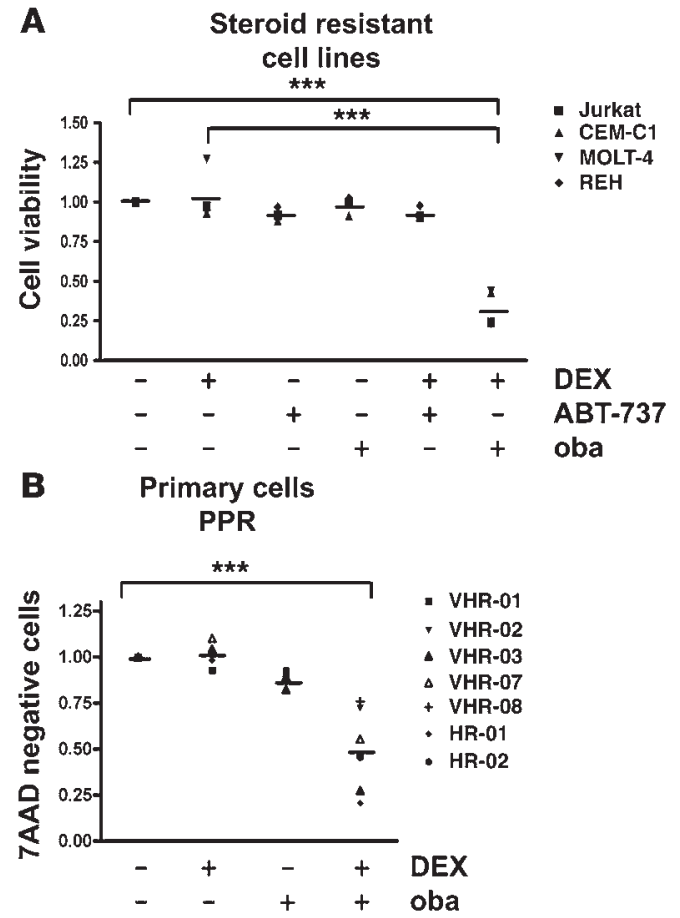

Steroid sensitive cell lines

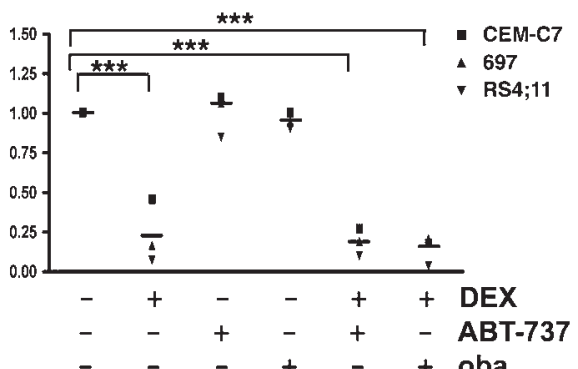

Primary cells PGR

$\star * *$

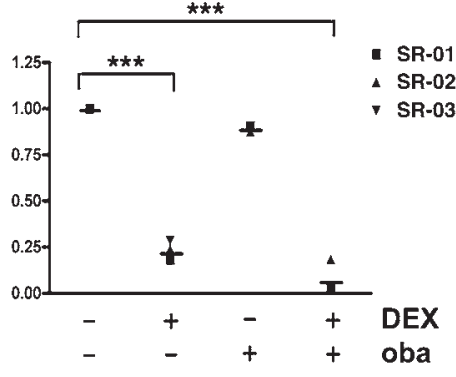

Figure 1

Obatoclax resensitizes GC-resistant ALL cells to dexamethasone. Combination experiments were performed with subcytotoxic concentrations $\left(10 \% \mathrm{IC}_{50}\right)$ of obatoclax (oba) or ABT-737 and $1 \mu \mathrm{M}$ dexamethasone (DEX), and values were normalized to cells treated with vehicle control. (A) ALL cell lines were treated for 72 hours as indicated. Cell viability was determined by the MTT assay. (B) Primary ALL cells from 7 PPR patients with VHR-ALL and 3 prednisone-good-responder (PGR) patients were treated as indicated for 72 hours. Cell viability was assessed by flow cytometry using 7AAD. ${ }^{* *} P<0.05$.

family members can engage in cross-talk between the apoptotic and autophagic pathways, as they were show to associate with the autophagy regulator beclin-1 $(11,14,15)$. When caspase-dependent apoptosis was blocked, a cell death mechanism that required autophagy was mediated via the receptor-interacting protein 1 (RIP-1) (12). RIP-1 is a central kinase that is associated with death receptor-induced signalling complexes to modulate the switch between survival and death under stress conditions (16). Under defective apoptotic conditions, RIP-1 kinase activity was shown to mediate an alternative cell death pathway that may represent a form of programmed necrosis, also called necroptosis $(17,18)$. Kinase activity of RIP- 1 was shown to be dispensable for induction of cell survival signalling via NF-kB activation or death receptor-mediated apoptosis $(17,19)$.

Here we show that a subcytotoxic concentration of obatoclax effectively restored the response to dexamethasone in GC-resistant ALL by triggering a nonapoptotic cell death pathway. Subcytotoxic concentrations of obatoclax induced disruption of beclin- 1 from MCL-1 and the combination of dexamethasone with obatoclax was associated with inhibition of mammalian target of rapamycin (mTOR) activity, providing a possible mechanism for autophagy induction. Obatoclax also conferred clinically relevant broad chemosensitization in multidrug-resistant primary cells from VHRALL patients. We provide genetic and pharmacologic evidence to show that sensitization to dexamethasone occurs via autophagydependent necroptotic cell death, while sensitization to other cytotoxic agents was dependent on apoptosis. In a xenograft model, using GC-resistant cells from refractory ALL patients, combina- tion treatment with dexamethasone and obatoclax reduced leukemia progression significantly. Collectively, our data provide a strong rationale for a rapid clinical translation of this approach.

\section{Results}

Obatoclax resensitizes GC-resistant ALL to dexamethasone. Because MCL-1 has been proposed to act as a central modulator of steroid resistance in ALL $(4,5)$, we first compared the effects of 2 small molecules with different selectivity toward MCL-1, ABT-737, and obatoclax in GC-resistant ALL cell lines. ABT-737 is a small molecule BH3 mimetic with selectivity for BCL-2 and BCL- $\mathrm{X}_{\mathrm{L}}$ (20). Resistance to ABT-737 has been attributed to its inability to target MCL-1 (21-23). While both compounds displayed strong cytotoxicity across a panel of ALL cell lines (Supplemental Table 1; supplemental material available online with this article; doi:10.1172/JCI39987DS1), subcytotoxic doses of obatoclax but not ABT-737 resensitized GC-resistant cell lines to dexamethasone (Figure 1A and Supplemental Figure 1). We confirmed single-agent activity for obatoclax in VHR-ALL cells (Supplemental Table 2). Low-dose obatoclax restored sensitivity to dexamethasone in cells from all 7 poor-prednisone-responder (PPR) VHR-ALL patients tested (Figure 1B and Supplemental Figure 2). In contrast, no effect on steroid sensitivity using low doses of ABT-737 or obatoclax was evident in steroid-sensitive cells (Figure 1A). Unexpectedly, the pan-caspase inhibitor zVAD.fmk did not interfere with the steroid-sensitizing effect of obatoclax in GC-resistant cells (Figure 2A), whereas zVAD.fmk blocked the cytotoxic effect of dexamethasone with or without obatoclax in GC-sensi- 
A

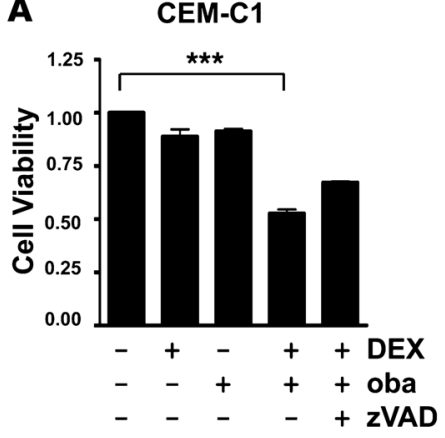

B
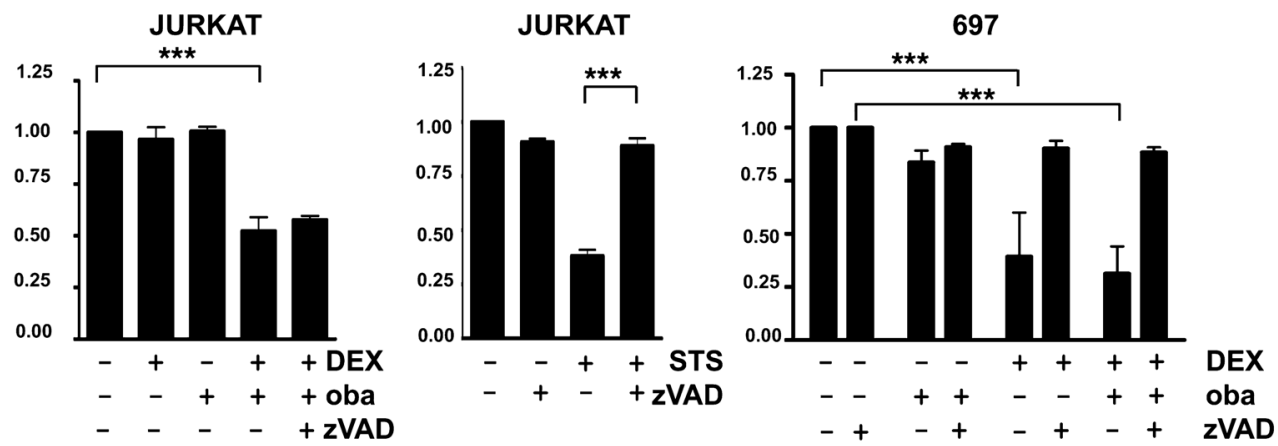

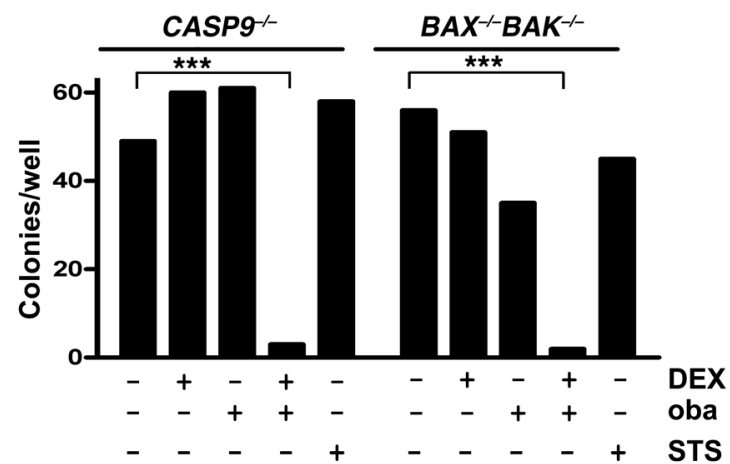

C

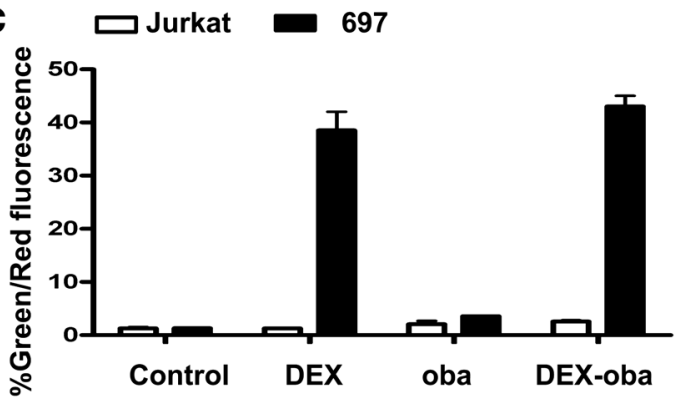

VHR-01 SR-02

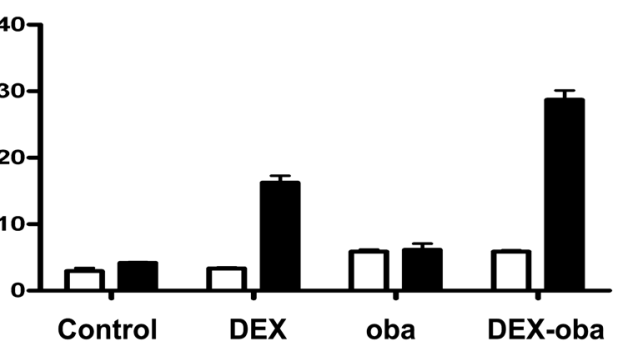

D
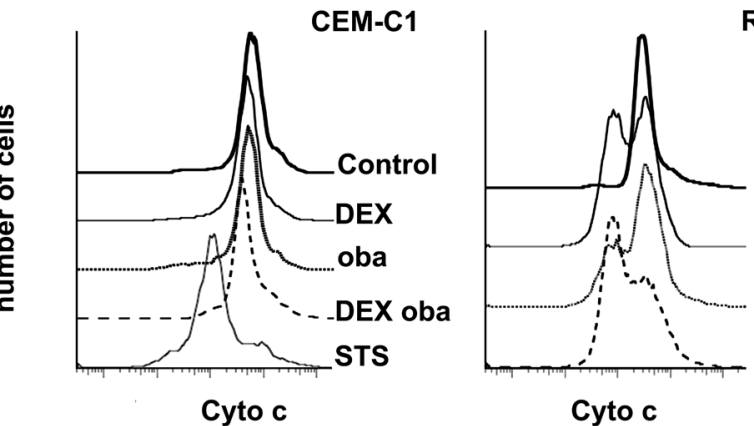

RS4;11

Cyto c

Figure 2

Obatoclax resensitizes GC-resistant ALL cells to dexamethasone without activation of mitochondrial apoptosis. (A) ALL cells were treated as indicated for 48 hours, for controls STS or ZVAD.fmk ( $80 \mathrm{nM}$ ) was used, and cell viability was assessed with the MTT assay. 697 cells served as GC-sensitive control. (B) Jurkat $C A S P 9^{-/-}$and $B A X^{-1-} B A K^{-1-}$ cells were treated for 72 hours as indicated, and clonogenic survival was assessed after incubation in methylcellulose for 7 days. (C) Percentages of cells with JC-1 monomers, corresponding to a loss of the mitochondrial potential, are shown for GC-resistant CEM-C1 and GC-sensitive CEM-C7 cells and in samples from PGR and PPR patients after treatment as indicated for 16 hours. (D) Cytochrome $c$ release was induced in steroid-sensitive RS4;11 cells but not in the resistant CEM-C1 cell line upon treatment with dexamethasone or dexamethasone and obatoclax. STS was used as positive control. Cytochrome $c$ release was detected by flow cytometry. ${ }^{* \star} P<0.05$.

tive cells and in control experiments when cell death was induced by staurosporine (STS). Using clonogenic assays, we confirmed that obatoclax was equally effective in ALL cells that are devoid of caspase- 9 or BAX and BAK, when compared with parental cell lines (Figure 2B and Supplemental Figure 3). Furthermore, obatoclax-mediated GC sensitization resulted in neither the restoration of mitochondrial membrane depolarization (Figure 2C) nor an increase in cytochrome $c$ release when compared with the effect 
A

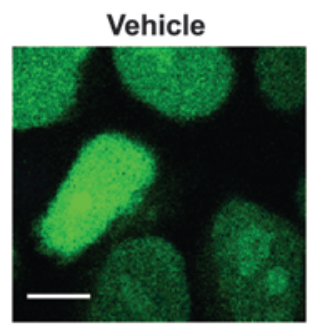

B

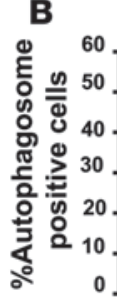

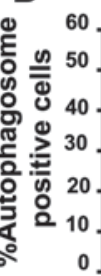

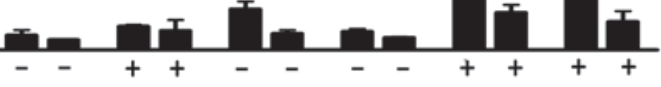

- $-\quad-\quad+$

$-\quad--$ -

$-+-+-$

D
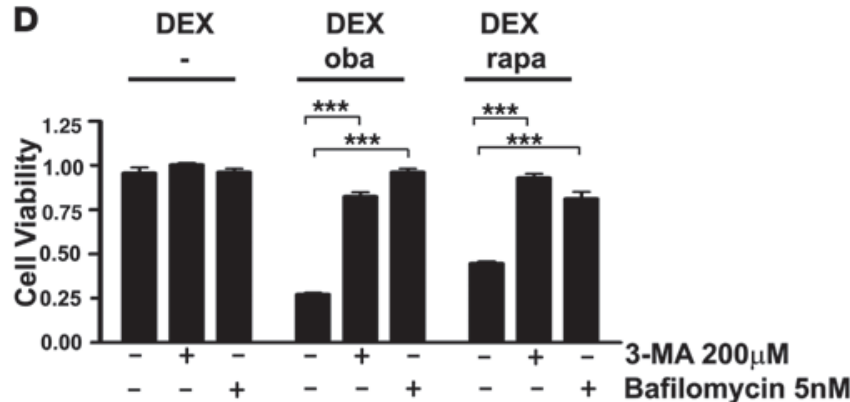

Rapamycin

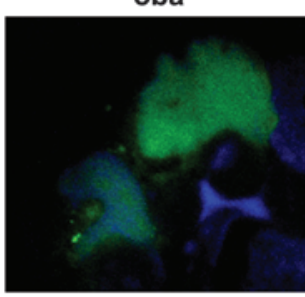

ᄎᄎ夫

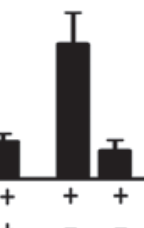

$+\quad+\quad-\quad-\quad+\quad+$

3-MA $200 \mu \mathrm{M}$

DEX $1 \mu \mathrm{M}$

oba 50nM

$+$

DEX

+ Bafilomycin $5 \mathrm{nM}$
DEX oba

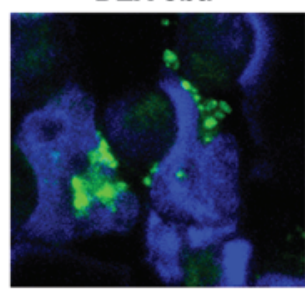

C

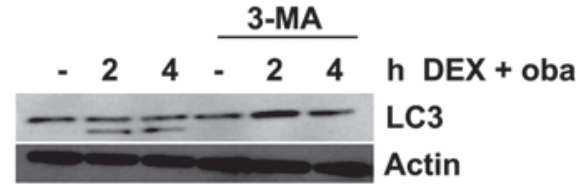

- DEX oba

DEX

oba

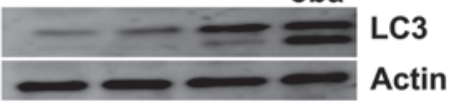

E
F

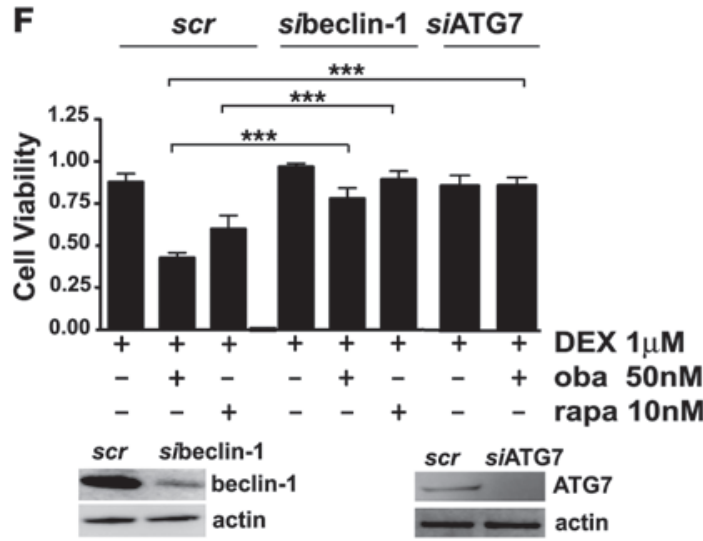

G

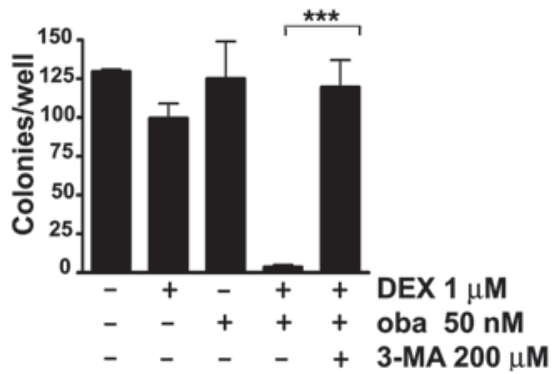

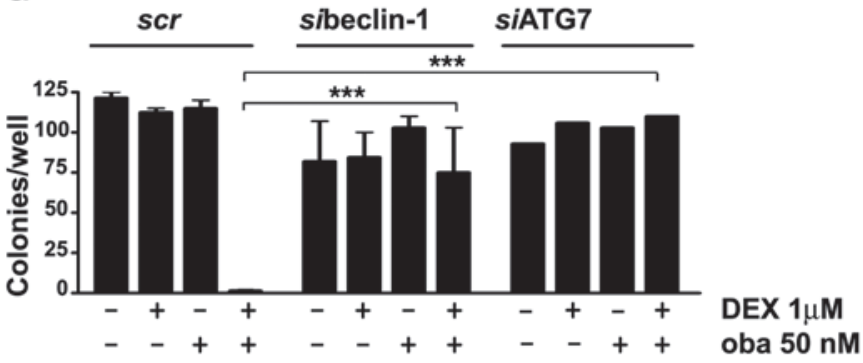

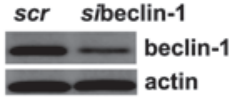

scr siATG7

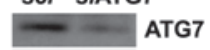

ATG7

Figure 3

Obatoclax induces autophagy in GC-resistant ALL cells. (A) After transient transfection with GFP-LC3, Jurkat cells were treated for 4 hours as indicated. The characteristic punctuate staining pattern indicative of autophagosome formation was detected by confocal microscopy in cells treated with dexamethasone and obatoclax or rapamycin (rapa). Scale bar: $10 \mu \mathrm{m}$. (B) Quantitation of autophagosome-positive cells. The data represent mean \pm SD of 2 independent experiments, counting 200 cells each. (C) Detection of endogenous LC3-II by Western blot analysis. Jurkat cells were treated with obatoclax and dexamethasone for the indicated time points in the presence or absence of 3-MA and after 24 hours of treatment as indicated. (D) Inhibition of autophagy by 3-MA or bafilomycin impaired sensitization to dexamethasone by $10 \mathrm{nM}$ rapamycin or obatoclax $\left(10 \% I_{50}\right)$. Cell viability was assessed by MTT. (E) Treatment with obatoclax and dexamethasone inhibited clonogenic survival of Jurkat cells. 3-MA rescued GC-resistant cells from cell death induced by combination treatment. Cells were treated for 72 hours with compounds, and clonogenic survival in methylcellulose was assessed after washing and incubation for 7 days. (F) Downregulation of beclin-1 or ATG7 using siRNA (si) impaired the resensitization of Jurkat cells to dexamethasone by obatoclax or rapamycin compared with scrambled (scr) controls. Cell viability was assessed by MTT. Efficiency of downregulation at 48 hours was analyzed by Western analysis. (G) Downregulation of beclin-1 and ATG7 in Jurkat cells protected cells from obatoclax- and dexamethasone-induced cell death in the clonogenic assay. ${ }^{\star \star \star} P<0.05$. 

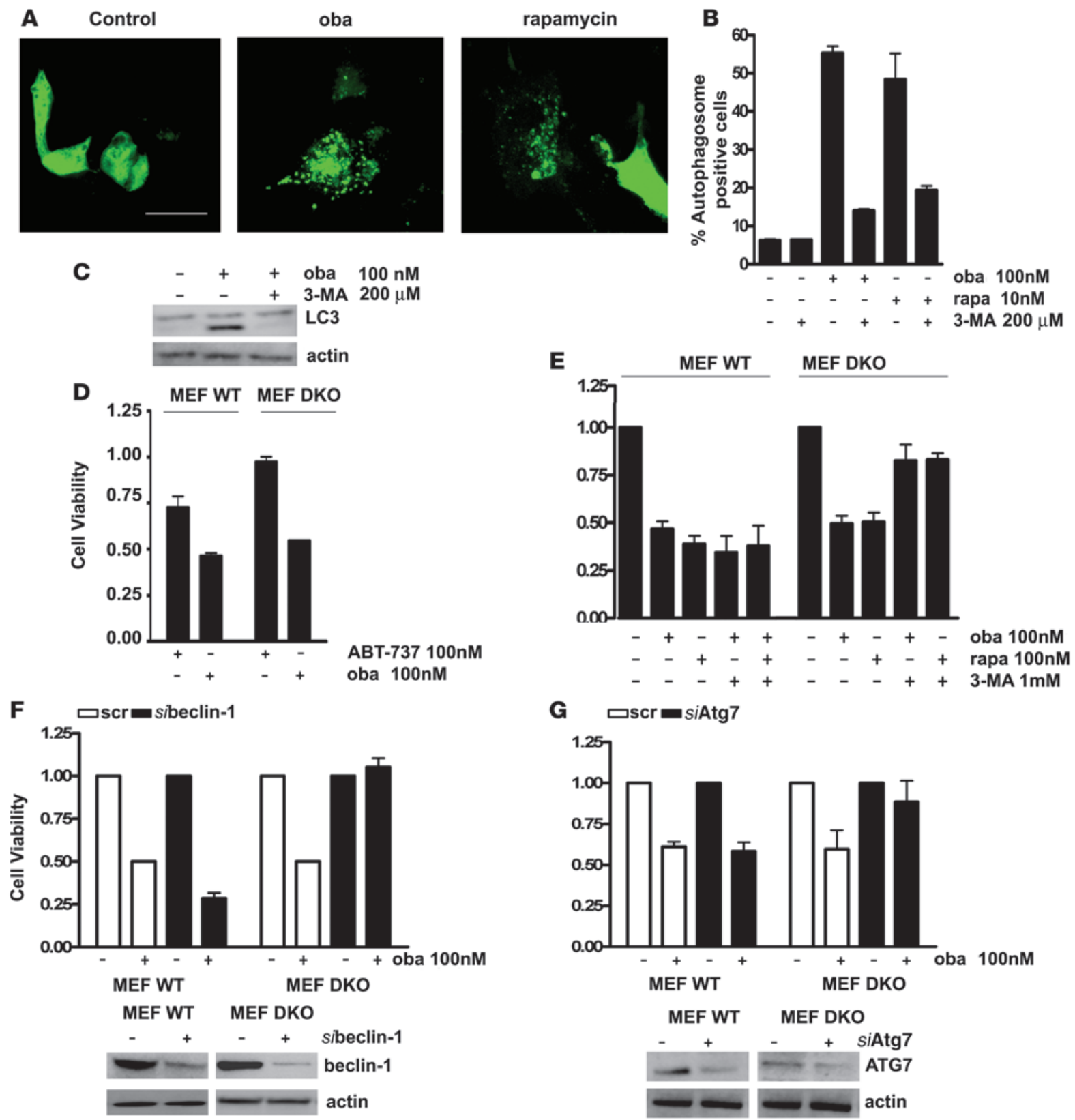

\section{Figure 4}

Obatoclax and rapamycin induce autophagic cell death in Bax ${ }^{-1-} \mathrm{Bak}^{-1}$ MEFs. (A) Bax ${ }^{-1-} \mathrm{Bak}^{-1-}$ MEFs transiently expressing the autophagy marker GFP-LC3 were treated with vehicle, obatoclax $(100 \mathrm{nM})$, or rapamycin $(10 \mathrm{nM})$ for 4 hours. Autophagosome formation was monitored by confocal microscopy. Scale bar: $20 \mu \mathrm{m}$. (B) Quantitation of autophagosome-positive cells. The data represent mean \pm SD of 2 independent experiments, counting 200 cells each. (C) Treatment of $\mathrm{Bax}^{-1-} \mathrm{Bak}^{-1}$ MEFs with obatoclax induced generation of endogenous LC3-II as detected by Western analysis, an effect which was blocked by 3-MA treatment. (D) WT or Bax ${ }^{-1-}$ Bak $^{-1-}$ (DKO) MEFs were incubated for 48 hours with obatoclax or ABT-737, and cell viability assessed by the MTT assay. (E) Inhibition of autophagy by 3-MA rescued Bax ${ }^{-1-}$ Bak $^{-1-}$ MEFs but not WT MEFs from cell death induced

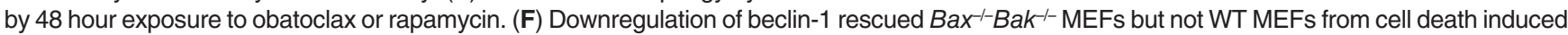
by obatoclax as evaluated by the MTT assay. Efficiency of downregulation was determined by Western blot analysis. (G) Downregulation of ATG7 rendered Bax ${ }^{-1-} \mathrm{Bak}^{-1-}$ MEFs but not WT MEFs resistant to obatoclax treatment. Efficiency of downregulation was assessed by Western blotting.

of dexamethasone in GC-sensitive cells (Figure 2D). In line with these findings, caspase- 9 was not activated and caspase- 3 was only marginally activated in steroid-resistant cells treated with the combination of obatoclax and dexamethasone (Supplemental Figure 4). Collectively, our results indicate that the combination of obatoclax and dexamethasone triggers a cell death mechanism that is independent of the apoptotic function of caspases in steroid-resistant leukemia.

Induction of autophagy is essential for steroid sensitization in ALL cells. Because an autophagy-dependent cell death pathway has been 


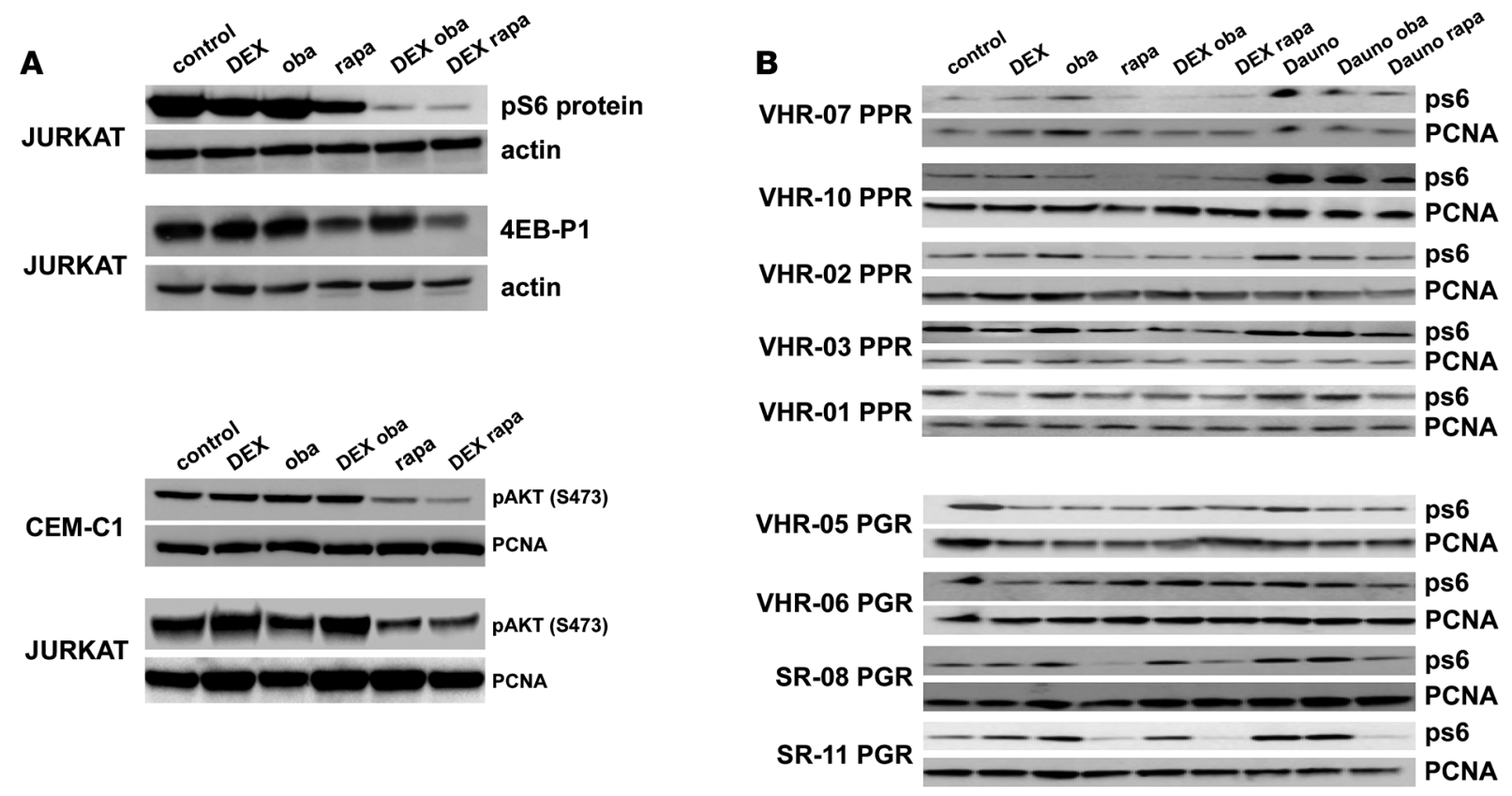

Figure 5

Combination treatment with dexamethasone and obatoclax leads to inhibition of mTOR. (A) Jurkat cells were treated for 6 hours as indicated, and phosphorylation of the mTOR targets, S6 protein and 4EB-P1, was assessed. Combination of dexamethasone with either obatoclax or rapamycin resulted in inhibition of mTOR activity (top panel). Rapamycin alone and in combination with dexamethasone, but not obatoclax alone or with dexamethasone, decreased the phosphorylation of AKT at Ser473 in Jurkat and CEM-C1 cells (bottom panel). (B) In primary cells from PPR patients with VHR-ALL, treatment with dexamethasone and obatoclax or rapamycin resulted in a decrease of S6 protein phosphorylation. In contrast, in cells from prednisone-good-responder patients, S6 protein was not dephosphorylated after combination treatment.

described in cell line models in which apoptosis is prevented $(11,24)$, we evaluated next whether autophagy was required for GC sensitization by obatoclax. Indeed, autophagosome formation was evident in GC-resistant cells after 4 hours of exposure to low-dose obatoclax in combination with dexamethasone, which correlated with generation of endogenous LC3-II (Figure 3, A-C). Because rapamycin is known to be an inducer of autophagy (25), we asked whether the GC-sensitizing effect reported recently for rapamycin (5) could also be dependent on autophagy. As we expected, steroid sensitization by rapamycin was associated with hyperautophagic features (Figure 3, $\mathrm{A}$ and $\mathrm{B})$. Obatoclax or rapamycin alone only marginally induced autophagosome formation and generation of LC3-II (Figure 3, A-C). Preincubation with the autophagy inhibitor 3-methyladenine (3-MA) abolished autophagosome formation, generation of LC3-II, and GC resensitization by obatoclax and rapamycin (Figure 3, B-D). Both bafilomycin, a late-stage inhibitor of autophagolysosome formation (25), and downregulation of the essential autophagy genes beclin-1 (BECN1) and ATG-7 (ATG7) by RNA interference blocked GC-resensitization by obatoclax and rapamycin (Figure 3, D-G). These results were confirmed in an independent cell line (Supplemental Figure 5). Clonogenic assays demonstrate that both addition of 3-MA and downregulation of beclin-1 prevented the cytotoxic effect of lowdose obatoclax combined with dexamethasone, since clonogenic growth was comparable to that in untreated controls (Figure 3, F and G). In GC-sensitive ALL cells, inhibition of autophagy by 3-MA only marginally restored GC resistance, despite small amounts of detectable LC3 lipidation (Supplemental Figure 6). These findings implicate autophagy as part of a common mechanism for GC resensitization by obatoclax and rapamycin.
To determine whether obatoclax triggers the autophagy pathway as a general response to blockade of mitochondrial apoptosis, we studied the drug's effects on $\mathrm{Bax}{ }^{-/-} \mathrm{Bak}^{-/-}$mouse embryo fibroblasts (MEFs), which exhibit resistance to a broad range of apoptotic stimuli (26). Like rapamycin, obatoclax induced autophagy in $\mathrm{Bax}^{-/-} \mathrm{Bak}^{-/-}$MEFs (Figure 4, A-C), as reflected by autophagosome formation (Figure 4, A and B) and generation of LC3-II (Figure 4C). Obatoclax was cytotoxic to WT and $\mathrm{Bax}^{-1-} \mathrm{Ba} k^{-/-} \mathrm{MEFs}$, whereas ABT-737's activity was restricted to WT MEFs (Figure 4D). The death response was blocked by 3-MA (Figure 4E) and downregulation of beclin-1 or ATG-7 (Figure 4, F and G). Taken together, these results indicate that the cytotoxic effects of obatoclax and rapamycin derive from induction of autophagy when mitochondrial apoptosis is blocked.

Modulation of the GC response by obatoclax is associated with inhibition of mTOR. A key regulator of cell fate decisions, including regulation of autophagy, is the kinase mTOR (27). Inhibition of mTOR by rapamycin resensitizes steroid-resistant ALL cells to dexamethasone (5), but the role of autophagy has not been investigated in this study. Since dexamethasone has been shown to inhibit mTOR in other cellular systems (28), we investigated the effect of dexamethasone and obatoclax treatment on mTOR activity. Alone dexamethasone slightly induced a decrease of S6 protein phosphorylation (Figure 5A). Treatment with obatoclax or rapamycin and dexamethasone resulted in a sharp decrease of the phosphorylation of the mTOR targets S6 protein and 4EB-P1 (Figure 5A). Interestingly, rapamycin but not obatoclax, either alone or in combination with dexamethasone, induced dephosphorylation of AKT at Ser473 (Figure 5A). Combination of obatoclax or rapamycin and dexamethasone also resulted in 
A

$$
\text { IP: anti-MCL-1 }
$$

beclin-1 -+ obatoclax

MCL-1

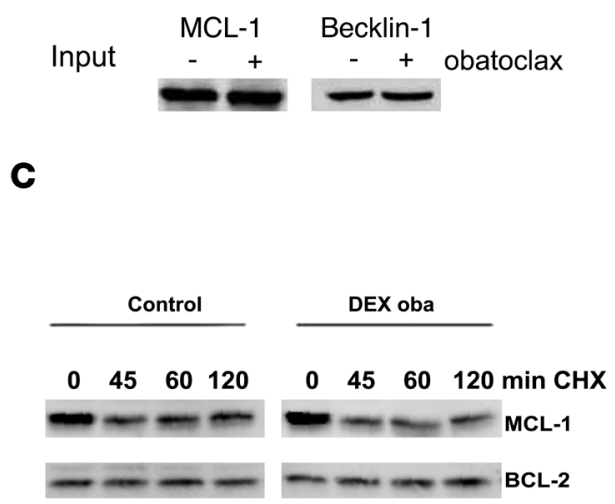

B

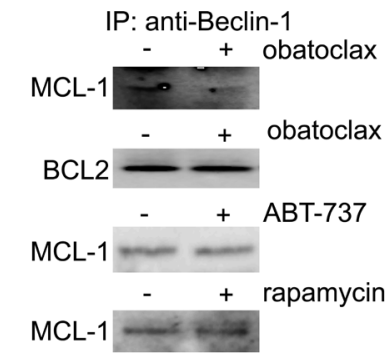

IP: anti-MCL-1
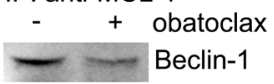

- + obatoclax

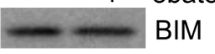

- $\quad+$ obatoclax
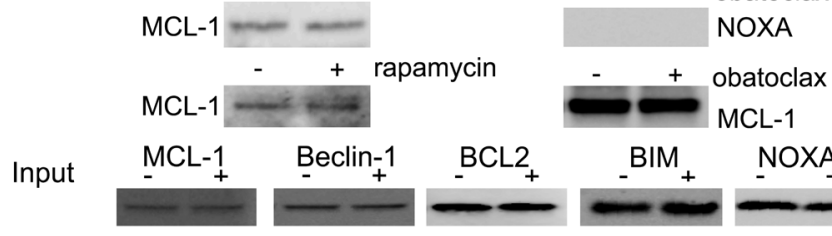

D

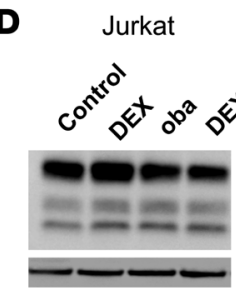

$\mathrm{RS} 4 ; 11$

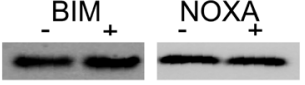

697

$30+0^{\circ}$
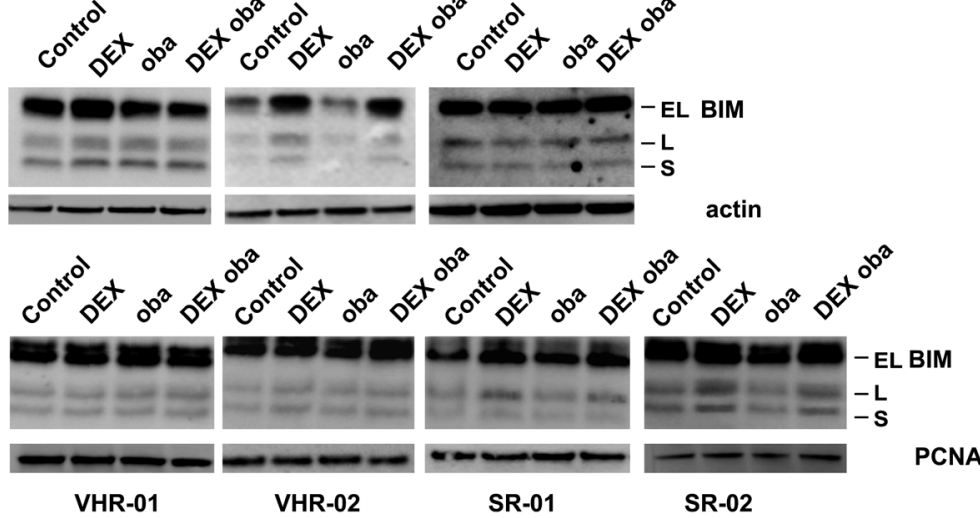

Figure 6

Obatoclax induces disruption of a complex of MCL-1 and beclin-1. (A) FLAG-tagged beclin-1 was overexpressed in 293T cells, and MCL-1 immunoprecipitates were analyzed for the presence of beclin-1, with or without obatoclax treatment for 3 hours. (B) Jurkat cells were treated with $100 \mathrm{nM}$ obatoclax, $30 \mathrm{nM} \mathrm{ABT-737,} \mathrm{or} 10 \mathrm{nM}$ rapamycin for 3 hours, and MCL-1 or beclin-1 immunoprecipitates were analyzed for the presence of proteins as indicated. Low-dose obatoclax disrupted the interaction between beclin-1 and MCL-1, while interactions between BCL2 and beclin-1 were unaffected. Likewise, ABT-737 or rapamycin did not affect complexes between beclin-1 and MCL-1 or BCL2. (C) MCL-1 levels were assessed after treatment with vehicle or the combination of dexamethasone and obatoclax at indicated time points, with or without cycloheximide (CHX). Western blot analyses revealed that MCL-1 levels were unaffected by combination treatment. (D) BIM induction was assessed by Western blot in steroid-resistant (Jurkat, VHR-01, and VHR-02) and steroid-sensitive (RS4;11, 697, SR-01, and SR-02) cell lines and primary cells treated with DEX $(1 \mu \mathrm{M})$, obatoclax $(100 \mathrm{nM})$, or the combination for 24 hours.

dephosphorylation of S6 protein in primary ALL cells from steroidresistant high risk ALL patients (Figure 5B, left). In contrast, cells from steroid-sensitive patients did not show dephosphorylation of S6 protein after treatment with obatoclax and dexamethasone (Figure 5B, right). Our experiments suggest that in the context of GC resistance, exposure to dexamethasone could cooperate with exposure to obatoclax to decrease mTOR activity. This finding will be exploited to develop correlative markers of biological response to in vivo treatment, for example, using phospho-flow cytometry to monitor the phosphorylation status of S6 protein in leukemia cells.

Obatoclax disrupts a complex between beclin-1 and MCL-1. The antiapoptotic BCL-2 family member MCL-1 was proposed to act as a modulator of GC resistance (5). Because the autophagy regulator beclin-1 was described to interact with antiapoptotic BCL-2 family members, including MCL-1 $(11,14,15)$, we next tested the effect of obatoclax on the interaction of beclin-1 with MCL-1. Subcytotoxic concentrations of obatoclax resulted in a markedly decreased detection of MCL-1 that coimmunoprecipitated with overexpressed, epitope-tagged beclin-1 in 293T cells (Figure 6A). We confirmed this result by performing coimmunoprecipitation experiments of endogenously expressed proteins in ALL cells
(Figure 6B). Again, in presence of subcytotoxic concentrations of obatoclax, less beclin-1 was shown to coimmunoprecipitate with MCL-1, and inversely, less MCL-1 was pulled down together with beclin-1. Obatoclax did not modify interactions between beclin-1 and BCL-2. Interestingly, incubation with rapamycin or ABT-737 did not affect the complex between beclin-1 and MCL-1. As expected from the reported MCL-1 interaction spectrum (29, 30), BIM was detectable by coimmunoprecipitation from steroid-resistant ALL cells, but binding of BIM to MCL-1 was not altered by treatment with low-dose obatoclax. The BH3-only protein NOXA, which was shown to interact preferentially with MCL-1, did not bind to MCL-1 in this experimental setting (Figure 6B).

Since MCL-1 can be subjected to a high protein turnover (31), we evaluated the effect of combination treatment with obatoclax and dexamethasone on MCL-1 protein levels. In the presence of cycloheximide, MCL-1 levels rapidly decreased after 45 minutes of incubation, independent of the presence of obatoclax and dexamethasone (Figure 6C). Absence of proapoptotic BIM induction by dexamethasone has been described in selected cases of steroid-resistant ALL (32). We did not detect any changes in BIM expression levels to correlate with the restored response to dexamethasone in combination with obatoclax 
A Control
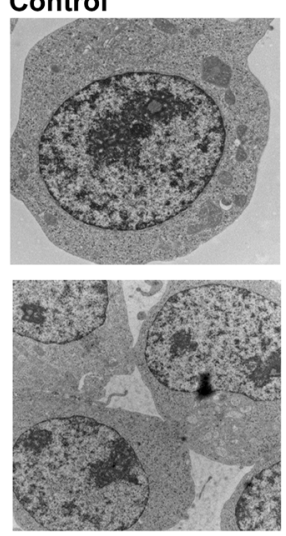

B Control

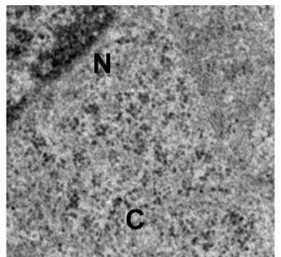

C

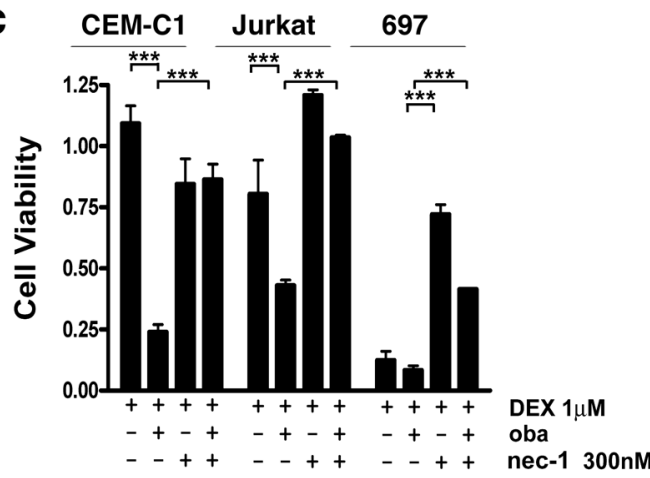

$\mathbf{F}$

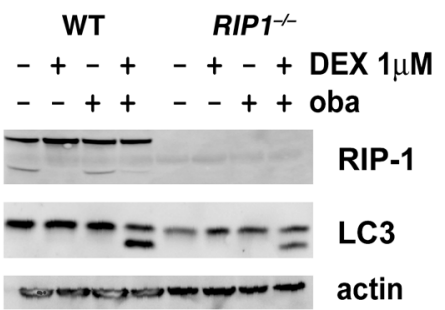

\section{DEX oba}
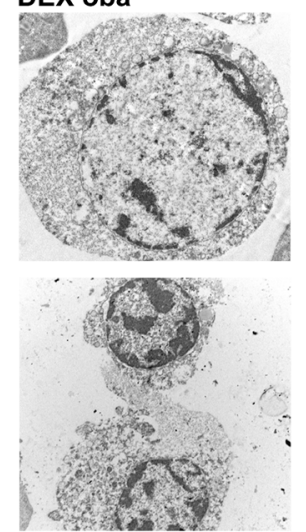

DEX oba

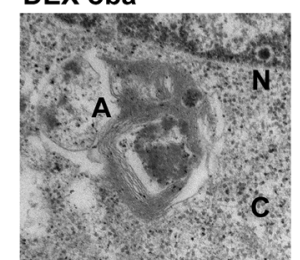

697

nec-1 300nM
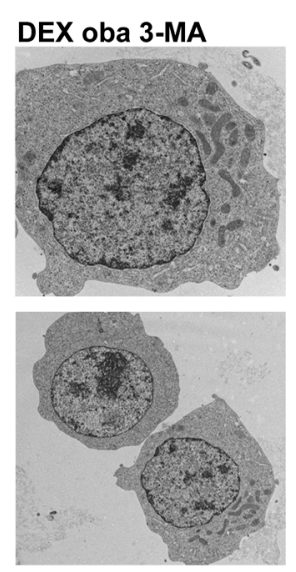

Control

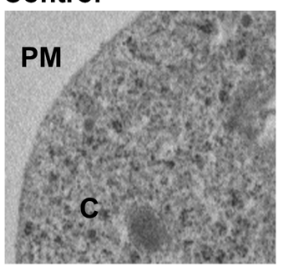

D $\underline{\text { RIP1-- }}$ WT

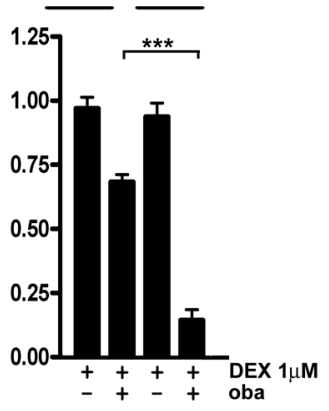

G

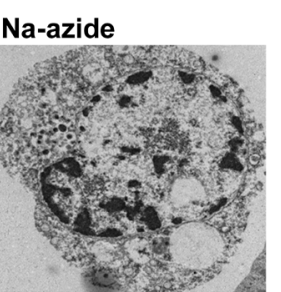

Trail
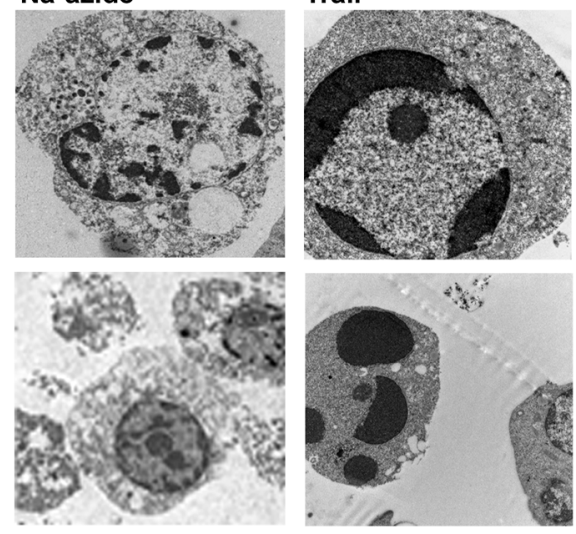

DEX oba

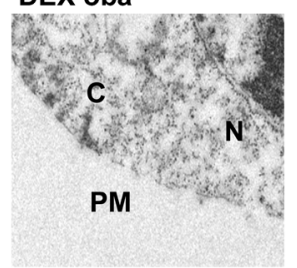

E scr

siCYLD-1

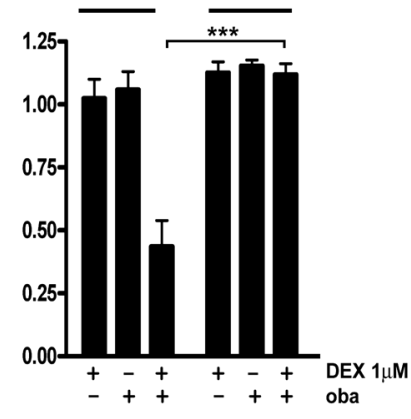

scr siCYLD

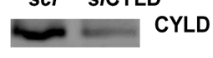

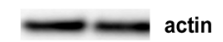

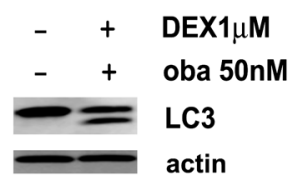

\section{Figure 7}

RIP-1 kinase activity is essential for obatoclax-mediated GC sensitization. (A) Electron microscopy images reveal features of necroptotic cell death after treatment for 72 hours with obatoclax and dexamethasone. No condensed chromatin was detectable, while Trail treatment induces condensed apoptotic nuclei (top panel) (original magnification, $\times 7,100$ ). Cells treated with obatoclax and dexamethasone exhibited disintegrated plasma membranes, which was recapitulated by Na-azide. Trail treatment leaved membranes intact (bottom panel) (original magnification, $\times 5,400$ ). (B) A more detailed view of the same experiment. An autophagosome formation with characteristic double membrane structures was detected in the cytoplasm. The plasma membrane was disrupted in cells treated with obatoclax and dexamethasone. N, nucleus; C, cytoplasm; A, autophagosome; PM, plasma membrane (original magnification, $\times 15,000)$. (C) In steroid-resistant (CEM-C1 and Jurkat) and steroid-sensitive (697) cells treated for 72 hours with dexamethasone $(1 \mu \mathrm{M})$ and obatoclax $\left(10 \% \mathrm{IC}_{50}\right)$, with or without the necroptosis inhibitor nec-1 $(300 \mathrm{nM})$, nec-1 restored steroid resistance as assessed by the MTT assay. (D) Jurkat $R I P 1^{-1-}$ cells were less sensitive to the double treatment for 72 hours with dexamethasone $(1 \mathrm{mM})$ and obatoclax $\left(10 \% \mathrm{IC}_{50}\right)$ compared with WT Jurkat cells. Cell viability was assessed by the MTT assay. (E) Downregulation of CYLD rescued Jurkat cells from cell death induced by combination treatment for 72 hours with dexamethasone (1 $\mu \mathrm{M})$ and obatoclax $\left(10 \%, \mathrm{IC}_{50}\right)$. Efficiency of downregulation was assessed by Western blot analysis after 48 hours. (F) LC3-II generation occurred in Jurkat RIP1-I- cells and in WT cells after 8 hours of treatment with dexamethasone and obatoclax. (G) Treatment with nec-1 did not inhibit LC3-II generation induced by treatment with obatoclax and dexamethasone in Jurkat cells. ${ }^{* \star *} P<0.05$. 

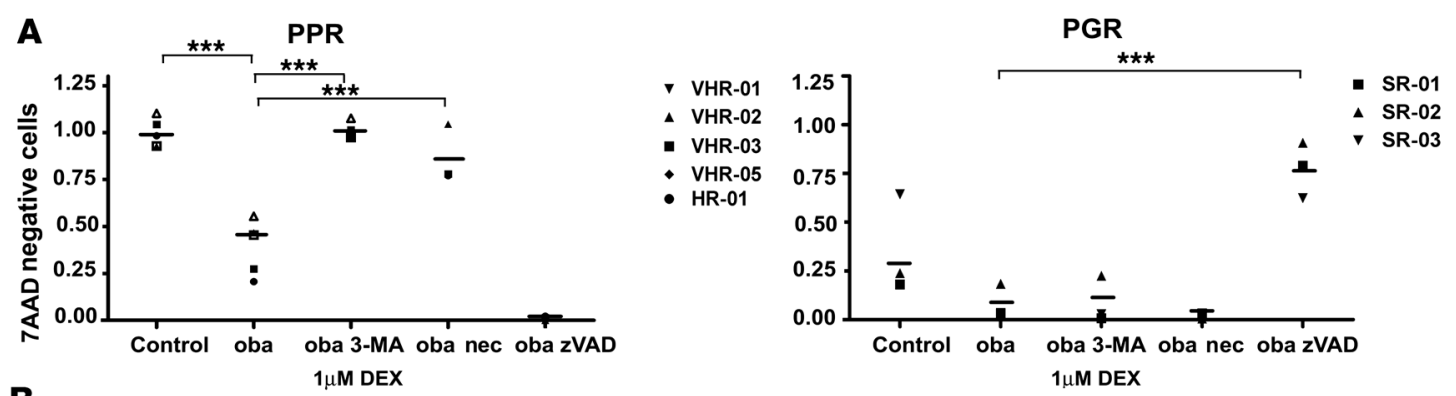

B
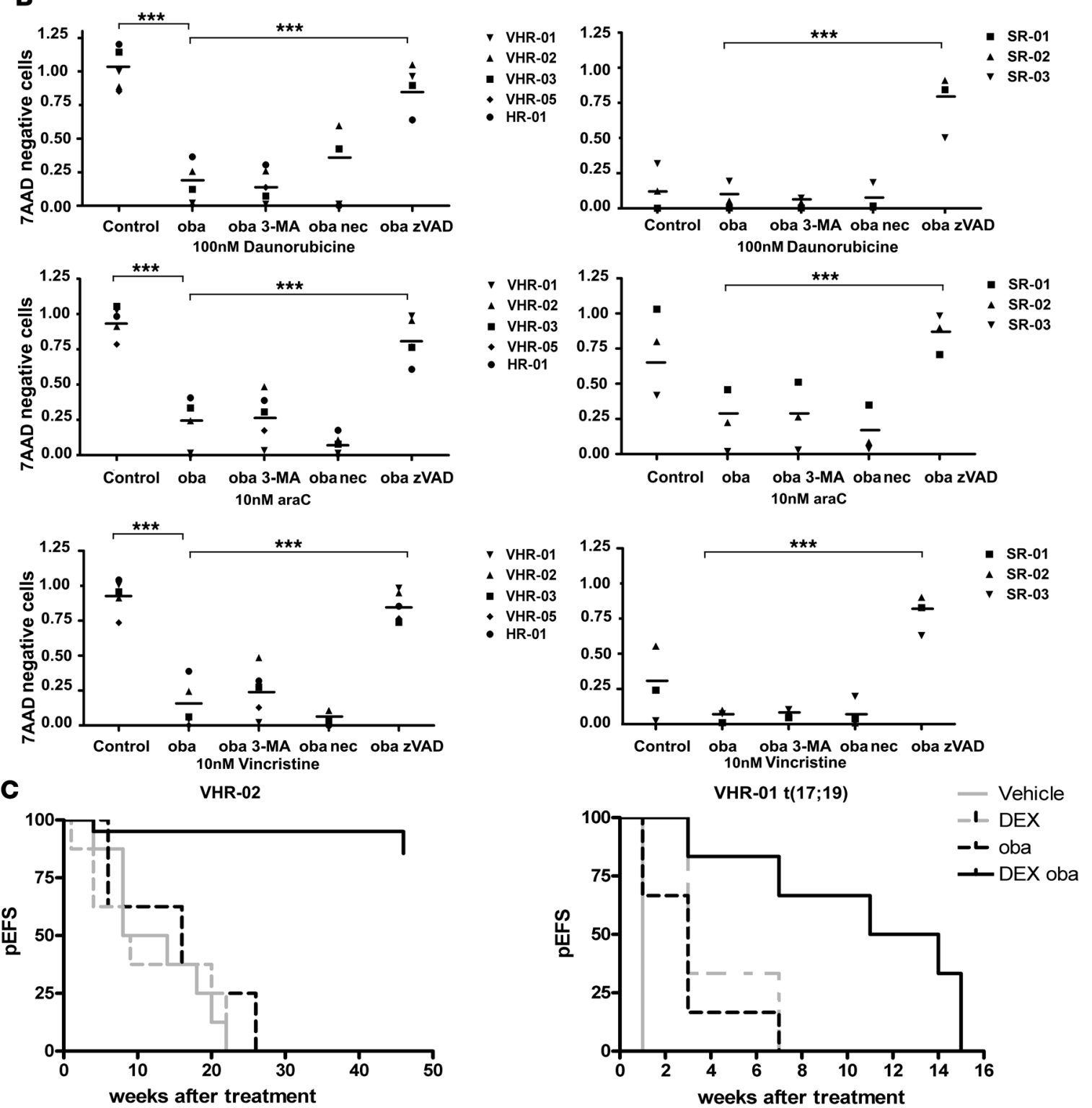


\section{Figure 8}

Obatoclax displays a strong chemosensitizing activity in multidrug-resistant primary ALL cells from poor risk patients. (A) Primary ALL cells from 5 PPR patients and 3 prednisone-good-responder patients were cocultured with hTERT-immortalized bone marrow stroma cells and treated with either dexamethasone $(1 \mu \mathrm{M})$ alone or in combination with obatoclax $\left(10 \% \mathrm{IC}_{50}\right)$ in the presence or absence of 3-MA, nec-1, or zVAD.fmk for 72 hours. Cell viability was assessed by 7AAD staining and flow cytometry. Data are shown as mean \pm SD of 2 independent experiments. In combination experiments, values were normalized to cells treated with compounds and/or inhibitors alone at indicated dose. (B) Primary ALL cells from $4 \mathrm{VHR}, 1$ high risk (HR), and 3 standard risk (SR) patients were cocultured with $\mathrm{hTERT}$-immortalized bone marrow stroma cells and treated with either daunorubicin, vincristine, or cytarabine (araC) alone or in combination with obatoclax $\left(10 \% \mathrm{IC}_{50}\right)$ in the presence or absence of 3-MA, nec-1, or zVAD.fmk for 72 hours. Cell viability was assessed by 7AAD staining and flow cytometry. Data are shown as mean $\pm \mathrm{SD}$ of 2 independent experiments. ${ }^{* * *} P<0.05$ (A and B). (C) Percentage of event-free survival ( $p E F S$ ) of NSG mice after xenotransplantation with primary cells from 2 PPR patients with VHR-ALL (VHR-02, $n=8$; patient VHR-01, $n=6$; Supplemental Table 2) and treatment for 3 weeks with either vehicle, dexamethasone, obatoclax, or the combination. $P<0.02$ for dexamethasone and obatoclax versus vehicle, dexamethasone, or obatoclax alone for both xenograft experiments.

in GC-resistant ALL cells (Figure 6D). Interestingly, induction of BIM was not detectable consistently in steroid-sensitive cells (Figure 6D). While in RS4;11 cells, BIM was readily induced by dexamethasone, neither 697 cells nor primary ALL cell samples from steroid-resistant patients showed induction of this proapoptotic BH3-only protein after dexamethasone treatment (Figure 6D). In cells from steroid sensitive patients, a small increase in the BIM-L and BIM-S but not BIM-EL isoforms was detectable. These data indicate that steroid resensitization by obatoclax is not due to induction of BIM.

To obtain further insight into the role of MCL-1 for the mechanism of action of obatoclax, we modulated MCL-1 expression using RNA interference. A reduction of MCL-1 protein levels resulted in partial resensitization to dexamethasone but, surprisingly, prevented any additional chemosensitization by the combination with obatoclax (Supplemental Results and Supplemental Figure 7). Partial resensitization to dexamethasone was mediated via induction of apoptosis, which was blocked using the pan-caspase inhibitor zVAD.fmk. Inhibition of autophagy with 3-MA did not affect the resensitization effect of MCL-1 knockdown to dexamethasone, even in presence of obatoclax. Instead, caspase- 9 activation was detected, which correlated with a caspase-dependent decrease of beclin-1. Thus, it is possible that induction of apoptosis overrides induction of autophagy when MCL-1 is downregulated, which limits the interpretation of these experiments. Taken together, our data indicate that disruption of the beclin-MCL-1 interaction is part of the mechanism triggered by obatoclax and suggest an involvement of MCL-1 in the regulation of an autophagy-dependent cell death pathway, in addition to its role as antiapoptotic regulator in leukemia cells.

Combination of obatoclax and dexamethasone triggers RIP-1 kinasedependent necroptosis in GC-resistant ALL. To better assess the nature of cell death occurring with obatoclax and dexamethasone, we proceeded with imaging by transmission electron microscopy. Indeed, autophagosome formation was evident after treatment with obatoclax and dexamethasone, which was completely inhibited by incubation with 3-MA (Figure 7, A and B). Cells displayed features consistent with a nonapoptotic cell death mechanism after expo- sure to obatoclax and dexamethasone. Condensation of chromatin, a hallmark of classical apoptosis, was incomplete (Figure 7A, top row), which contrasts with the marked chromatin condensation after treatment with TRAIL, which induces death receptor-dependent apoptosis in ALL cells (33). A characteristic feature of dead cells was the disintegration of the plasma membrane (Figure 7B), which is a hallmark of necroptosis (34). Increasing evidence suggests that a programmed necrotic cell death pathway can be triggered in the context of impaired apoptotic effectors, and this is dependent on RIP-1 kinase activity $(12,17)$. RIP-1 functions at the intersection of cell survival and cell death signalling $(35,36)$. Therefore, we tested whether inhibition of RIP-1 with necrostatin-1 (nec-1; ref. 17) would interfere with steroid sensitization by obatoclax. Indeed, steroid-resistant ALL cells treated with nec-1 were refractory to obatoclax-induced dexamethasone resensitization (Figure 7C). Nec-1 also reduced the cytotoxic response to dexamethasone, alone or in combination with low-dose obatoclax in steroid-sensitive ALL cells (Figure 7C). Interestingly, nec-1 inhibited induction of BIM in steroid-sensitive cells that showed BIM induction after dexamethasone (Supplemental Figure 8). Consistent with a central role for RIP-1 for the response to GC drugs, RIP-1-deficient ALL cells (37) were resistant to obatoclax-mediated GC resensitization (Figure 7D).

The deubiquitinase cylindromatosis (turban tumor syndrome) (CYLD) is a direct regulator of RIP-1 kinase activity (38). Knocking down CYLD expression showed that CYLD is required for RIP-1dependent cell death induction by combination of dexamethasone and obatoclax (Figure 7E). Our results are consistent with the current model, which proposes that the deubiquinated form of RIP-1 is mediating cell death signals (35). An important finding is that induction of autophagy could be detected early, within 4 hours of treatment, and that generation of LC3-II was neither inhibited in RIP-1-deficient ALL cells (Figure 7F) nor in nec-1-treated ALL cells (Figure 7G). Taken together, these results demonstrate that the combination of obatoclax and dexamethasone triggers a nonapoptotic cell death pathway, which shares the central features of necroptosis, including the characteristic morphology of cell death shown by electron microscopy, and a strict dependence on RIP-1 kinase activity and RIP-1 deubiquitinase CYLD. Moreover, our data indicate that the autophagic process is triggered upstream of RIP-1 and does not occur as a consequence of necroptosis.

Obatoclax is a broad chemosensitizer in multidrug-resistant primary $A L L$ cells, but activation of autophagy-dependent cell death is specific to the combination with dexamethasone. To validate our results in primary cells, we tested pretreatment samples from PPR patients with precursor $B$ cell ALL, who were further characterized by the molecular persistence of leukemic cells under intensified conventional multiagent chemotherapeutic treatment and predicted to have a very high risk of relapse based on their poor in vivo response to chemotherapy (Supplemental Table 2). Indeed, low-dose obatoclax resensitized GC-resistant ALL cells to dexamethasone in mesenchymal stroma cell (MSC) cocultures, which was completely abolished using 3-MA, implicating autophagy as an essential mechanism in primary refractory ALL cells also (Figure 8A). At this dosage, obatoclax alone induces less than $5 \%$ cell death. Importantly, 3-MA did not interfere with the cytotoxic effect of treatment with dexamethasone, alone or combined with obatoclax, on primary ALL cells from GC-sensitive patients (Figure 8A), indicating that autophagy was not required for the death response to GC drugs in GC-sensitive cells. Consistent with our results in ALL cell lines, nec-1 abolished the GC-sensitizing activity of combination treatment in steroid-resistant primary 
VHR-ALL cells. In contrast, blocking caspases with zVAD.fmk did not inhibit steroid resensitization in primary GC-resistant ALL cells, while the response to dexamethasone was inhibited by zVAD. fmk treatment in cells from steroid-sensitive ALL patients.

Current treatment protocols include a steroid window followed by multidrug chemotherapy, including cytotoxic agents such as daunorubicin, vincristine, and cytarabine. Low-dose obatoclax resensitized primary refractory ALL cells to all 3 drugs, while the sensitivity of cells from sensitive patients was not increased further. Interestingly, 3-MA did not restore resistance to these cytotoxic agents, indicating that induction of autophagy after treatment with obatoclax is specific for GC resensitization (Figure 8B). In contrast, blocking caspases using zVAD.fmk completely inhibited the resensitizing effect of low-dose obatoclax in combinations with daunorubicin, vincristine, and cytarabine, both in cells from very high risk and from standard risk patients. These results support the notion that autophagy-dependent necroptosis is specifically triggered with GCs in a GC-resistant context. Moreover, obatoclax could serve as a chemosensitizer for established multidrug regimens for ALL treatment, given its broad potential to restore the apoptotic response with non-GC antileukemic drugs.

Combination of obatoclax and dexamethasone is effective in a leukemia xenograft model, using cells from refractory ALL patients. To test the in vivo efficacy of obatoclax in GC resensitization, we transplanted primary ALL cells from 2 VHR-PPR patients into NOD/SCID/ IL2r $\gamma^{\text {null }}$ (NSG) mice (39). When 1\% human leukemic cells were detected in mouse peripheral blood, we treated mouse cohorts with vehicle, obatoclax $(5 \mathrm{mg} / \mathrm{kg} / \mathrm{d})$, dexamethasone $(5 \mu \mathrm{g} / \mathrm{kg} / \mathrm{d})$, or both for 3 weeks. In vehicle-treated animals, progression to leukemia (>10\% peripheral blasts) was observed in a median time of 11 weeks for animals xenografted with cells from patient VHR-02 and 1 week for animals xenografted with cells from patient VHR-01. All mice xenografted with cells from patient VHR-02 and treated with single-agent dexamethasone or obatoclax had to be sacrificed due to leukemia progression, while only 2 out of 8 animals treated with the combination of obatoclax and dexamethasone progressed to leukemia over the 50-week observation period. A significant delay in leukemia progression was achieved by combination treatment with dexamethasone and obatoclax when cells from VHR-01 were used $(P<0.02$ for combination treatment versus vehicle or single agent treatment). This patient showed the recurrent translocation $\mathrm{t}(17 ; 19)(\mathrm{q} 22 ; \mathrm{p} 13)$, which is virtually always associated with early relapse (40) (Figure 8C). This result suggests that GC sensitization of VHR-PPR ALL cells can be achieved in vivo, providing a strong rationale to explore the therapeutic potential of combined dexamethasone and obatoclax clinically.

\section{Discussion}

Here we describe a pharmacological approach that we believe to be new to specifically bypass the apoptotic blockade to chemotherapy in multidrug-resistant ALL. Subcytotoxic concentrations of the BCL-2 antagonist obatoclax restored the response to dexamethasone by inducing a nonapoptotic cell death pathway. The activation of autophagy-dependent cell death in cells that are resistant to the apoptotic stimuli by dexamethasone is reminiscent of observations that were reported using experimental systems with defined genetic defects of key regulators of the intrinsic and extrinsic apoptotic response. In $\mathrm{Bax}{ }^{-1-} \mathrm{Bak}^{-/-} \mathrm{MEF}$ and BCL-2overexpressing MEFs, cell death triggered by etoposide or STS was dependent on autophagy genes beclin-1 and ATG-5 (11). As also reported by others $(6,8)$, obatoclax was cytotoxic for $B a x^{-/-} B a k^{-/-}$ cell lines, which we demonstrate to be critically dependent on the autophagy pathway. Similarly, genetic or pharmacologic interference with caspase 8 or death receptor signalling can result in autophagy-dependent cell death with necroptotic features $(12,18$, 41). In lymphoid cells, this may constitute an alternative mechanism to control abnormal cellular proliferation in the absence of a normal apoptotic response. In activated $T$ cells from mice with caspase- 8 or FADD deficiency, autophagic signalling was required to induce RIP-1-dependent necroptotic cell death (41). We here show that this mechanism of cell death can be activated in GC-resistant leukemia to restore the response to dexamethasone.

Autophagy has been recognized as an important regulatory mechanism of cell fate decisions. While it is clear that autophagy can have a protective function at times of cellular stress, the contribution of autophagy to the execution of programmed cell death is a subject of controversy (13). Our data demonstrate that autophagic signalling is an integral part of the cell death mechanism when the response to dexamethasone is restored with obatoclax or rapamycin. Both inhibitors that interfere early (3-MA) or late (bafilomycin) with the autophagic process and knockdown of genes that are essential for autophagy, BECN1 and ATG7, prevented resensitization to dexamethasone completely. Combination of dexamethasone and obatoclax inhibited clonogenic growth of GC-resistant ALL cells, and electron microscopy imaging unequivocally showed rapid induction of necrotic cell death with autophagic features. There is clear evidence that in cancer, autophagy is not necessarily a protective feature. For example, BECN1 was shown to act as a haploinsufficient tumor suppressor gene $(42,43)$, with increased frequency of spontaneous neoplasia, including lymphomas in beclin-1-haploinsufficient mice. The genes that are essential for the autophagic machinery are highly conserved and required in several autophagy-dependent cellular processes (44). Several studies link autophagy genes to programmed cell death. In the central nervous system for example, ATG7 deficiency protected neurons from caspase-dependent and caspase-independent cell death after hypoxic/ischemic brain injury (45). In human glioblastoma, knockdown of the autophagy genes ATG1 or ATG5 prevented the cytotoxic effect of cannabinoids, which induce autophagydependent cell death via an mTORC1-dependent pathway (46). Autophagy was also shown to regulate programmed cell death during development. The steroid hormone ecdysone triggered autophagy-dependent cell death during morphogenesis of salivary glands from the larval stage to the adult stage in Drosophila. This cell death pathway was independent of caspase activity (47), providing compelling evidence for the modulation of an autophagic cell death pathway via steroid hormone signalling in normal development. Similarly, autophagy is required for programmed cell death in the midgut during Drosophila metamorphosis, which provides additional evidence for specific regulation of cell death by autophagy, even in presence of an intact apoptotic machinery (48). Combination treatment of obatoclax with GC drugs but not with other cytotoxic agents induced autophagy-dependent cell death in resistant ALL. This raises the question of whether autophagy is also required for the effect of dexamethasone in GC-sensitive ALL. A recent report described increased autophagy after dexamethasone treatment in GC-sensitive ALL cell lines, but cell death was associated with apoptotic features and knockdown of beclin-1 resulted only in partial rescue of this effect (49). We also observed a partial reduction of dexamethasone cytotoxicity using 3-MA (Supplemen- 
tal Figure 6) or nec-1 (Figure 7C) in a subset of GC-sensitive cell lines. We could, however, not detect an effect of 3-MA in primary ALL cells, in which caspase-dependent cell death prevailed. Our results identify autophagy as an early and limiting step to steroid sensitization by obatoclax in GC-resistant cells and underscore the importance of understanding the cellular context when designing strategies to target autophagy for cancer treatment.

There is clear evidence for hyperactivation of AKT (50) and mTOR (5) in GC-resistant ALL. Because mTOR is implicated in the control of autophagy in different settings (27), hyperactive AKT-mTOR signalling could prevent induction of autophagy in resistant disease. We hypothesized that GC-resistant ALL cells could therefore be primed for mTOR-controled autophagy. Consistent with this idea, we found that induction of autophagic cell death by the combination of dexamethasone and obatoclax resulted in marked reduction in phosphorylation of the mTOR target $\mathrm{S} 6$ protein in resistant cells. The mechanisms by which obatoclax or rapamycin potentiate the effect of dexamethasone on mTOR appear to be different. Indeed combination of dexamethasone with rapamycin, but not with obatoclax, resulted in a marked decrease in phosphorylation of AKT on Ser473, consistent with recent finding showing that $\mathrm{mTOR}$ can also act upstream of AKT (51). Modulation of mTOR target phosphorylation was only seen when obatoclax was combined with dexamethasone, but not with other cytotoxic agents, suggesting that dexamethasone exposure contributes to inhibition of mTOR. Indeed, exposure to dexamethasone was reported to be associated with repression of mTOR signalling in myoblast cell lines and lymphoid cells $(28,52)$. The importance of mTOR for the control of autophagy is also underscored by the results of a comprehensive screen using a chemical compound library in order to identify new pharmacologic inducers of autophagy, in which proautophagic activity of candidate molecules was always associated with decreased phosphorylation of mTOR targets (53). Extensive studies will be required to dissect the primary signalling events triggered by the combination of obatoclax and dexamethasone, as they may provide important clues about the mechanisms of drug resistance in ALL.

GC resistance does not appear to be associated with genetic or functional defects of the GC receptor in $\operatorname{ALL}(54,55)$. Dexamethasone was proposed to induce apoptosis by increasing the levels of the $\mathrm{BH} 3$-only proapoptotic protein BIM, which were markedly reduced in selected cases of GC-resistant ALL (32). However, in most cases tested, we could not detect induction of BIM by dexamethasone in GC-sensitive cell lines and primary ALL cells. Furthermore, we did not detect increased levels of BIM, decreased MCL-1 protein levels, or increased MCL-1 protein turnover associated with steroid sensitization. Instead, we found, by immunoprecipitation of endogenous proteins, subcytoxic concentrations of obatoclax to result in the disruption of MCL-1 with beclin-1 in ALL cells. This suggests the possibility that MCL-1 could control induction of autophagy via beclin-1, as it was proposed for other BCL-2 family members recently $(14,15)$. Interestingly, this effect was not seen in cells treated with rapamycin, again pointing out that the target mechanism of obatoclax is different. We recognize the fact that this effect could also result from indirect mechanisms on the protein complex, including MCL-1 and beclin-1. Furthermore, functional experiments modulating MCL-1 expression levels were not conclusive. Knockdown of MCL-1 resulted in moderate activation of apoptosis and partial sensitization to dexamethasone that was caspase dependent but prevented complete resensitization to dexamethasone by obatoclax and autophagic cell death. Activation of apoptosis is possibly overriding induction of autophagy in this context. In support of this hypothesis, we detected activation of caspase-9 after downregulation of MCL-1, with caspase-9-dependent cleavage of beclin-1. This provides a possible mechanism to prevent autophagy induction when apoptosis is activated. Taken together, steroid modulation with low-dose obatoclax did not involve release of proapoptotic BCL-2 family proteins from MCL-1 but triggered autophagy-dependent cell death by a mechanism that required the presence of MCL-1.

In this context, activation of autophagy is required to induce necroptosis, a form of programmed necrosis that has been described to occur when apoptosis is abortive due to caspase inhibition $(16,56)$. Execution of necroptosis is dependent on the RIP-1 kinase (17). Our data demonstrate that RIP-1 activity is absolutely required for steroid sensitization by obatoclax and by rapamycin. The cell death morphology that was documented by electron microscopy is consistent with the morphology reported for necroptosis $(12,56)$. Furthermore, the deubiquitinase cylindromatosis (CYLD), which has been shown to regulate RIP-1 (38), was functionally required for execution of necroptosis. This is consistent with experiments showing that RIP-1 kinase activity was required to trigger cell death and that ubiquitination of RIP-1 prevents cell death signalling $(16,19)$. RIP-1 and CYLD were included among the core genes that were identified in a functional siRNA screen for genes that were essential for necroptosis (18). To the best of our knowledge, we provide the first clinically relevant model with consistent validation in primary leukemia cells from patients with very high risk disease, in which the necroptotic pathway can be exploited to restore response to therapy. As such, this will constitute a very relevant experimental model to study the mechanisms of necroptosis in depth. Our results clearly imply a direct link between the autophagic pathway and RIP-1-mediated signalling events leading to necroptosis. Autophagy is triggered early and independent of RIP-1 kinase activity, indicating that it acts upstream of necroptotic signals. A number of studies identify RIP functionally as part of a complex with proteins of the death receptor pathway, such as FADD and caspase-8 (35). Experiments using mouse models indicate that autophagy and necroptosis could be linked via recruitment of components of the death receptor pathway to the membrane of autophagosomes (41). It is tempting to speculate that a similar mechanism is triggered in ALL cells upon costimulation with dexamethasone and obatoclax. Our findings warrant extensive biochemical follow-up studies to understand how RIP-1 is activated and how the autophagic machinery is connected to the necroptotic pathway.

Based on promising studies by others (32), we have established a leukemia xenograft model of de novo highly resistant ALL. The power of this approach resides in the possibility to select cases from relevant patient groups, starting from cryopreserved leftover diagnostic samples, from one of the largest cooperative trials for the treatment of childhood ALL. By focusing on cases with VHR-ALL by MRD, we also selected for patients that are most significantly resistant to prednisone in vivo, as defined by the reduction of leukemia cells in the peripheral blood after 1 week of prednisone monotherapy. Accordingly, ALL cells from these patients were completely resistant to dexamethasone and other chemotherapeutic agents in vitro. Low-dose obatoclax restored the response to dexamethasone, both in precursor B cell and $\mathrm{T}$ cell ALL cases. The durable remissions observed with 1 year follow-up, 
using the leukemia xenograft model, are indicative of strong antileukemic activity. Furthermore, the broad chemosensitizing effect of low-dose obatoclax in combination with daunorubicin, vincristine, and cytarabine in multidrug-resistant primary ALL cells provides a strong basis for further evaluation of obatoclax in combination with a multidrug regimen. The xenograft approach will be essential to validate this approach for heavily pretreated relapse and refractory patients. Our observation that isolated clones can emerge in clonogenic assays after treatment of a cell line with obatoclax and dexamethasone indicates that resistance to this approach may occur. Our established xenograft system will enable us to screen a larger number of ALL cases to verify whether resistance to this approach has to be expected. The identification of resistant cases would provide a model to establish markers that correlate with response or resistance. Based on current data, dynamic changes of mTOR activity with treatment represent a good candidate marker. Such knowledge will serve to optimize patient selection for clinical trials.

Taken together, our data support a model in which the apoptotic blockade in GC-resistant ALL cells can be overcome by activating an autophagy-dependent necroptotic cell death pathway. The characteristic necroptotic features by electron microscopy and the changes in the phosphorylation profile of $\mathrm{S} 6$ protein provide tools to assess the biological response to combination treatment with obatoclax and dexamethasone in patients in refractory ALL. Given the acceptable toxicity profile of obatoclax in clinical studies in adults with hematologic malignancies $(9,10)$, our study provides a compelling rationale for the evaluation of this new pharmacological strategy for the treatment of children with refractory and relapsed ALL.

\section{Methods}

Cell culture, reagents, and standard procedures. CEM-C7-14 and CEM-C1-15 cells (referred to CEM-C7 and CEM-C1 in the text) were provided by E.B. Thompson (University of Texas Medical Branch, Galveston, Texas), human bTERT-immortalized primary bone marrow MSCs (57) were provided by D. Campana (St Jude Children's Research Hospital, Memphis, Tennessee), caspase-9-deficient and retransfected Jurkat cells (58) as well as Bax/Bakdeficient Jurkat cells (59) were provided by K. Schulze-Osthoff (University of Tübingen, Germany), and parental and Bax/Bak-deficient MEFs (26) were provided by J.-C. Martinou (University of Geneva, Switzerland). Rip $1^{-/-}$cells were provided by B. Seed (Harvard Medical School, Boston, Massachusetts). Obatoclax was provided by J. Viallet and G. Shore (Gemin X, Malvern, Pennsylvania) and ABT-737 was provided by S. Elmore and S. Rosenberg (Abbott Laboratories, Chicago, Illinois).

Detailed procedures are described in Supplemental Methods. For RNA silencing experiments, $30 \mathrm{nM}$ beclin-1 (sc-29797, Santa Cruz Biotechnology Inc.) was used. pEGFP-LC3 was provided by M. Jäättelä (Danish Cancer Society, Copenhagen) and the FLAG-tagged beclin-1 construct by B. Levine (University of Texas Southwestern Medical School, Dallas). All constructs were transfected by nucleofection (Amaxa) using solution $V$ and Amaxa program
T-016 for CEM-C1, A-024 for CEM-C7 cells, and U-020 for MEFs. Transfection efficiency was controlled using the pEGFP plasmid (Clontech).

Patient samples. ALL cells were recovered from cryopreserved anonymized samples from patients who were enrolled in the ongoing ALL-BFM 2000 protocol and had given informed consent in accordance with the Declaration of Helsinki. Approval was obtained from the Institutional Review Board (IRB) of the Medical School Hannover and the local IRB for all participating centers in the trial ALL-BFM 2000. This approval extends to the use of leftover diagnostic material for add-on research projects, including those addressing basic biological questions.

In vitro drug response. In vitro drug response curves in cell lines were established with the MTT assay and were normalized to vehicle control. Primary patient samples were cocultured on hTERT-immortalized human MSCs, and drug response curves were analyzed with flow cytometry using propidium iodide.

Xenograft model. Xenograft experiments were approved by the veterinary office of the Canton of Zurich. In brief, primary ALL cells were recovered from cryopreserved samples and transplanted intrafemorally to NSG mice. Leukemia progression was monitored by flow cytometry with human CD45 and CD19 antibodies (AbD Serotec). ALL cells recovered from spleens of NSG mice after the first xenotransplantation were used for in vivo therapeutic trials. Randomized cohorts were treated with vehicle intramuscularly (i.m.): $3 \mathrm{mg} / \mathrm{kg} / \mathrm{d}$ obatoclax i.m., $5 \mathrm{mg} / \mathrm{g} / \mathrm{d}$ dexamethasone i.p., or the combination of dexamethasone i.p. and obatoclax i.m. for 5 days per week for 3 consecutive weeks.

Statistics. All experiments were performed 4 times, unless stated otherwise in the figure legends. Data are represented as mean $\pm \mathrm{SD}$. For in vitro experiments, Student's $t$ test (2-tailed) was used on triplicates. $P$ values of less than 0.05 were considered significant. Event-free survival (EFS) was defined as the time from the start of treatment until $10 \%$ of human leukemia cells were detectable in the mouse peripheral blood and was assessed by Kaplan-Meier analysis. Survival curves were analyzed with the log-rank test (GraphPad prism). $P$ values of less than 0.05 were considered significant.

\section{Acknowledgments}

We thank U. Luethi for assistance with electron microscopy and L. Walensky, I. Maillard, S. Krishnan, and V. Saha for helpful discussions and feedback on the manuscript. This work was supported by grants to J.-P. Bourquin from the Fondation pour la Recherche Cancer de l' Enfant, the Swiss Cancer League, the Swiss National Science Foundation, the Foundation for Research at the Medical Faculty, University of Zurich, the Julius Müller Foundation, and the Huggenberger-Bischoff Foundation.

Received for publication May 26, 2009, and accepted in revised form January 6, 2010.

Address correspondence to: Jean-Pierre Bourquin, Department of Oncology, University Children's Hospital Zurich, Steinwiesstrasse 75, CH-8032 Zurich, Switzerland. Phone: 41.44.266.7304; Fax: 41.44.266.7171; E-mail: jean-pierre.bourquin@kispi.uzh.ch.
1. Pui CH, Evans WE. Treatment of acute lymphoblastic leukemia. NEngl J Med. 2006;354(2):166-178.

2. Schrappe M. Evolution of BFM trials for childhood ALL. Ann Hematol. 2004;83(Suppl 1):S121-S123.

3. Schrauder A, et al. Prospective evaluation of MRDKinetics in 274 children with high-risk ALL treated in trial ALL-BFM 2000: Insights into development of resistance and impact on further refinement of treatment stratification strategies. Blood. 2007;110:585.
4. Holleman A, et al. Gene-expression patterns in drug-resistant acute lymphoblastic leukemia cells and response to treatment. $N$ Engl J Med. 2004;351(6):533-542.

5. Wei G, et al. Gene expression-based chemical genomics identifies rapamycin as a modulator of MCL1 and glucocorticoid resistance. Cancer Cell. 2006;10(4):331-342.

6. Nguyen M, et al. Small molecule obatoclax (GX15070) antagonizes MCL-1 and overcomes MCL-1- mediated resistance to apoptosis. Proc Natl Acad Sci U S A. 2007;104(49):19512-19517.

7. Perez-Galan P, Roue G, Villamor N, Campo E, Colomer D. The BH3-mimetic GX15-070 synergizes with bortezomib in mantle cell lymphoma by enhancing Noxa-mediated activation of Bak. Blood. 2007;109(10):4441-4449.

8. Konopleva M, et al. Mechanisms of antileukemic activity of the novel Bcl-2 homology domain-3 mimetic GX15-070 (obatoclax). Cancer Res. 2008; 
68(9):3413-3420

9. O'Brien SM, et al. Phase I study of obatoclax mesylate (GX15-070), a small molecule pan-Bcl-2 family antagonist, in patients with advanced chronic lymphocytic leukemia. Blood. 2009;113(2):299-305.

10. Schimmer AD, et al. A phase I study of the pan bcl2 family inhibitor obatoclax mesylate in patients with advanced hematologic malignancies. Clin Cancer Res. 2008;14(24):8295-8301.

11. Shimizu S, et al. Role of Bcl-2 family proteins in a non-apoptotic programmed cell death dependent on autophagy genes. Nat Cell Biol. 2004;6(12):1221-1228

12. Yu L, et al. Regulation of an ATG7-beclin 1 program of autophagic cell death by caspase-8. Science. 2004;304(5676):1500-1502

13. Kroemer G, Levine B. Autophagic cell death: the story of a misnomer. Nat Rev Mol Cell Biol. 2008;9(12):1004-1010.

14. Maiuri MC, et al. Functional and physical interaction between $\mathrm{Bcl}-\mathrm{X}(\mathrm{L})$ and a $\mathrm{BH} 3$-like domain in Beclin-1. EMBO J. 2007;26(10):2527-2539.

15. Pattingre $\mathrm{S}$, et al. Bcl-2 antiapoptotic proteins inhibit Beclin 1-dependent autophagy. Cell. 2005;122(6):927-939.

16. Holler N, et al. Fas triggers an alternative, caspase-8-independent cell death pathway using the kinase RIP as effector molecule. Nat Immunol. 2000;1(6):489-495.

17. Degterev A, et al. Identification of RIP1 kinase as a specific cellular target of necrostatins. Nat Chem Biol. 2008;4(5):313-321.

18. Hitomi J, et al. Identification of a molecular signaling network that regulates a cellular necrotic cell death pathway. Cell. 2008;135(7):1311-1323.

19. O’Donnell MA, Legarda-Addison D, Skountzos P, Yeh WC, Ting AT. Ubiquitination of RIP1 regulates an NF-kappaB-independent cell-death switch in TNF signaling. Curr Biol. 2007;17(5):418-424.

20. Oltersdorf T, et al. An inhibitor of Bcl-2 family proteins induces regression of solid tumours. Nature. 2005;435(7042):677-681.

21. Deng J, Carlson N, Takeyama K, Dal Cin P, Shipp $\mathrm{M}$, Letai A. BH3 profiling identifies three distinct classes of apoptotic blocks to predict response to ABT-737 and conventional chemotherapeutic agents. Cancer Cell. 2007;12(2):171-185.

22. Konopleva M, et al. Mechanisms of apoptosis sensitivity and resistance to the $\mathrm{BH} 3$ mimetic ABT-737 in acute myeloid leukemia. Cancer Cell. 2006;10(5):375-388.

23. van Delft MF, et al. The BH3 mimetic ABT-737 targets selective $\mathrm{Bcl}-2$ proteins and efficiently induces apoptosis via Bak/Bax if Mcl-1 is neutralized. Cancer Cell. 2006;10(5):389-399.

24. Shao Y, Gao Z, Marks PA, Jiang X. Apoptotic and autophagic cell death induced by histone deacetylase inhibitors. Proc Natl Acad Sci U S A. 2004;101(52):18030-18035

25. Luo S, Rubinsztein DC. Atg5 and Bcl-2 provide novel insights into the interplay between apoptosis and autophagy. Cell Death Differ. 2007;14(7):1247-1250.
26. Lindsten $\mathrm{T}$, et al. The combined functions of proapoptotic Bcl-2 family members bak and bax are essential for normal development of multiple tissues. Mol Cell. 2000;6(6):1389-1399.

27. Sarbassov DD, Ali SM, Sabatini DM. Growing roles for the mTOR pathway. Curr Opin Cell Biol. 2005;17(6):596-603.

28. Wang H, Kubica N, Ellisen LW, Jefferson LS, Kimball SR. Dexamethasone represses signaling through the mammalian target of rapamycin in muscle cells by enhancing expression of REDD1. J Biol Chem. 2006;281(51):39128-39134.

29. Certo M, et al. Mitochondria primed by death signals determine cellular addiction to antiapoptotic BCL-2 family members. Cancer Cell. 2006;9(5):351-365.

30. Chen L, et al. Differential targeting of prosurvival $\mathrm{Bcl}-2$ proteins by their $\mathrm{BH} 3$-only ligands allows complementary apoptotic function. Mol Cell. 2005;17(3):393-403.

31. Maurer U, Charvet C, Wagman AS, Dejardin E, Green DR. Glycogen synthase kinase-3 regulates mitochondrial outer membrane permeabilization and apoptosis by destabilization of MCL-1. Mol Cell. 2006;21(6):749-760.

32. Bachmann PS, Gorman R, Mackenzie KL, LutzeMann L, Lock RB. Dexamethasone resistance in B-cell precursor childhood acute lymphoblastic leukemia occurs downstream of ligand-induced nuclear translocation of the glucocorticoid receptor. Blood. 2005;105(6):2519-2526

33. Fakler M, et al. Small molecule XIAP inhibitors cooperate with TRAIL to induce apoptosis in childhood acute leukemia cells and overcome Bcl-2-mediated resistance. Blood. 2009;113(8):1710-1722.

34. Miao B, Degterev A. Methods to analyze cellular necroptosis. Methods Mol Biol. 2009;559:79-93.

35. Declercq W, Vanden Berghe T, Vandenabeele P. RIP kinases at the crossroads of cell death and survival. Cell. 2009;138(2):229-232.

36. Galluzzi L, Kroemer G. Necroptosis: a specialized pathway of programmed necrosis. Cell. 2008;135(7):1161-1163.

37. Ting AT, Pimentel-Muinos FX, Seed B. RIP mediates tumor necrosis factor receptor 1 activation of NF-kappaB but not Fas/APO-1-initiated apoptosis. EMBOJ. 1996;15(22):6189-6196.

38. Wright A, et al. Regulation of early wave of germ cell apoptosis and spermatogenesis by deubiquitinating enzyme CYLD. Dev Cell. 2007;13(5):705-716.

39. Shultz LD, et al. Human lymphoid and myeloid cell development in NOD/LtSz-scid IL2R gamma null mice engrafted with mobilized human hemopoietic stem cells. J Immunol. 2005;174(10):6477-6489.

40. Matsunaga T, et al. Regulation of annexin II by cytokine-initiated signaling pathways and E2AHLF oncoprotein. Blood. 2004;103(8):3185-3191.

41. Bell BD, et al. FADD and caspase- 8 control the outcome of autophagic signaling in proliferating $\mathrm{T}$ cells. Proc Natl Acad Sci U S A. 2008;105(43):16677-16682.

42. Qu X, et al. Promotion of tumorigenesis by heterozygous disruption of the beclin 1 autophagy gene. J Clin Invest. 2003;112(12):1809-1820.
43. Yue Z, Jin S, Yang C, Levine AJ, Heintz N. Beclin 1, an autophagy gene essential for early embryonic development, is a haploinsufficient tumor suppressor. Proc Natl Acad Sci U S A. 2003;100(25):15077-15082.

44. Levine B, Kroemer G. Autophagy in the pathogenesis of disease. Cell. 2008;132(1):27-42.

45 . Koike $M$, et al. Inhibition of autophagy prevents hippocampal pyramidal neuron death after hypoxic-ischemic injury. Am J Pathol. 2008;172(2):454-469.

46. Salazar M, et al. Cannabinoid action induces autophagy-mediated cell death through stimulation of ER stress in human glioma cells. JClin Invest. 2009;119(5):1359-1372.

47. Berry DL, Baehrecke EH. Growth arrest and autophagy are required for salivary gland cell degradation in Drosophila. Cell. 2007;131(6):1137-1148.

48. Denton D, et al. Autophagy, not apoptosis, is essential for midgut cell death in Drosophila. Curr Biol. 2009;19(20):1741-1746

49. Laane E, et al. Cell death induced by dexamethasone in lymphoid leukemia is mediated through initiation of autophagy. Cell Death Differ. 2009;16(7):1018-1029.

50. Bornhauser BC, et al. Low dose arsenic trioxide sensitizes glucocorticoid-resistant acute lymphoblastic leukemia cells to dexamethasone via an Akt-dependent pathway. Blood. 2007;110(6):2084-2091.

51. Guertin DA, Sabatini DM. Defining the role of mTOR in cancer. Cancer Cell. 2007;12(1):9-22.

52. Wang Z, Malone MH, Thomenius MJ, Zhong F, Xu F, Distelhorst CW. Dexamethasone-induced gene 2 (dig2) is a novel pro-survival stress gene induced rapidly by diverse apoptotic signals. J Biol Chem. 2003;278(29):27053-27058.

53. Balgi AD, et al. Screen for chemical modulators of autophagy reveals novel therapeutic inhibitors of mTORC1 signaling. PLoS ONE. 2009;4(9):e7124.

54. Bachmann PS, et al. Divergent mechanisms of glucocorticoid resistance in experimental models of pediatric acute lymphoblastic leukemia. Cancer Res. 2007;67(9):4482-4490.

55. Tissing WJ, et al. Glucocorticoid-induced glucocorticoid-receptor expression and promoter usage is not linked to glucocorticoid resistance in childhood ALL. Blood. 2006;108(3):1045-1049.

56. Degterev A, et al. Chemical inhibitor of nonapoptotic cell death with therapeutic potential for ischemic brain injury. Nat Chem Biol. 2005;1(2):112-119.

57. Iwamoto S, Mihara K, Downing JR, Pui CH, Campana D. Mesenchymal cells regulate the response of acute lymphoblastic leukemia cells to asparaginase. J Clin Invest. 2007;117(4):1049-1057.

58. Janssen K, Pohlmann S, Janicke RU, SchulzeOsthoff K, Fischer U. Apaf-1 and caspase-9 deficiency prevents apoptosis in a Bax-controlled pathway and promotes clonogenic survival during paclitaxel treatment. Blood. 2007;110(10):3662-3672

59. Samraj AK, Stroh C, Fischer U, Schulze-Osthoff K. The tyrosine kinase Lck is a positive regulator of the mitochondrial apoptosis pathway by controlling Bak expression. Oncogene. 2006;25(2):186-197. 